\title{
Functional Rehabilitation Criteria Required for a Safe Return to Active Duty in Military Personnel Following a Musculoskeletal Injury: A Scoping Review
}

\author{
Nadine Houghton, ${ }^{a}$ Jared Maynard, ${ }^{a}$ and Alice Aiken ${ }^{a}$
}

\begin{abstract}
The objective of this article is to assess the types of musculoskeletal (MSK) injuries commonly affecting military personnel and the outcome measures that may be used to predict a safe return to active duty post-injury. The key word-driven electronic search identified 190 articles initially. Thirty-one articles remained following application of inclusion and exclusion criteria. The United States published 27 of the 31 studies, most of which were retrospective reviews, case series, prospective cohort studies, and randomized controlled trials. Based on inclusion frequency, MSK injuries of the shoulder, back, knee, ankle, and foot are the most prevalent in military populations. Physical therapy interventions varied significantly even among similar injury types with return-to-duty rates varying from 2 to 100 per cent over three to 20.9 months, depending on intervention and injury type. Many varied outcome measures were used between studies to evaluate subjects. No concrete criteria currently exist to evaluate readiness for a safe return to duty following an MSK injury. More widespread use of standardized protocols for specific injuries and taking into consideration the physical requirements for each military occupational specialty will assist in determining the readiness of recovering soldiers to return to their full duties in the future.
\end{abstract}

Key words: active duty personnel, enlisted personnel, job re-entry, military personnel, military recruits, reserve personnel, return to active duty, return to duty, return to work

\section{RÉSUMÉ}

Objectifs : Faire l'étude des types de blessures musculosquelettiques qui affectent le personnel militaire et les résultats qui pourraient prédire un retour au service (RT) sécuritaire suite à la blessure. Design : Étude de délimitation de l'étendue. Source de données : Base de données électronique CINAHL, Medline, Embase et PEDro. Sélection de l'étude : La recherche effectuée avec les mots-clés a identifié 190 articles au premier passage. Suite à l'application des critères d'inclusion et d'exclusion, 31 articles ont été sélectionnés. Résultats : 27 des 31 articles ont été publiés aux États-Unis, dont la majorité consistait en des revues rétrospectives, réexamens de cas, études de cohortes prospectives et des essais cliniques randomisés. En se basant sur la fréquence d'inclusion les blessures musculosquelettiques de l'épaule, du dos, du genou, de la cheville et du pied sont les plus prévalent avec la population militaire. Les interventions physio thérapeutiques variaient grandement, même pour des blessures similaires, avec des taux de retour au service entre 2 et 100\% prenant entre 3 et 20.9 mois, tout dépendant des interventions et du type de blessure. Plusieurs indicateurs des résultats variés ont été utilisés entre chaque étude pour évaluer les sujets. Conclusion : Pour le moment, aucun critère n'existe pour évaluer un retour au service sécuritaire suite à une blessure musculosquelettique. L'utilisation des protocoles standards pour une blessure spécifique, ainsi que la prise en considération des exigences physiques de chaque spécialités de travail militaire aidera à déterminer la disponibilité opérationnelle d'un soldat qui se rétablit d'une blessure avant son retour au service.

Mots clés : personnel militaire, retour au service, retour au travail, personnel militaire actif, gradés et hommes de troupes, recrus militaires, membres de la réserve.

a School of Rehabilitation Therapy, Queen's University, Kingston, ON

Correspondence should be directed to Alice Aiken, aa5@queensu.ca 


\section{INTRODUCTION}

Active military service carries with it an inherently high level of physical demand, both while undergoing training and while deployed in operations. This physicality often leads to military personnel sustaining a variety of injuries over the course of their service career that may require time off for recovery before returning to active duty. Musculoskeletal (MSK) injuries have increased in the military and are one of the major causes of medical evacuation and battle attrition in recent wars. ${ }^{1,2}$ The variety of these types of injuries - traumatic, overuse, battle related, non-battle related - and the variability in physical demands between military roles often make it difficult to quantify the criteria needed to ensure a safe return to duty after an injury. This may be a reason why poor outcomes and low return-to-duty rates are reported in the literature. ${ }^{2}$ Guiding prevention and treatment protocols with better understanding of the tasks of soldiers, and injuries that may commonly occur, can help to maintain efficiency in the military and preserve the fighting force. ${ }^{1}$ The objective of this scoping review was to survey the current literature for indications of criteria leading to a safe return to active duty post-injury, to use this information to guide future research, and to inform best practice within military forces caring for injured personnel.

\section{METHODS}

This scoping review uses the methodology presented by Arksey and $\mathrm{O}^{\prime}$ Malley ${ }^{3}$ with additional recommendations made by Levac, Colquhoun, and O'Brien. ${ }^{4}$ This combined approach uses five steps to scan a vast body of literature and answer a research question. These steps are:

(1) identify the research question;

(2) identify relevant studies;

(3) determine and apply inclusion/exclusion criteria;

(4) chart the data; and

(5) summarize and report the results.

For Step 1, the research question was: In military personnel returning to active duty post-injury, what functional criteria achieved through rehabilitation must be met to ensure a safe return to duty?

For Step 2, the following electronic databases were searched: CINAHL, Medline, Embase, and PEDro. The keywords used in the search were military personnel to specify the target population as well as one word from the following set to specify the target outcome for the population: return to work, return to duty, return to active duty. Embase required a larger set of first search terms (military personnel, active duty personnel, enlisted personnel, military recruits, reserve personnel) and substituted job re-entry for return to work in the second set of search terms. The search of PEDro used only military personnel as a first search term. In total, the databases that were searched yielded 190 results. Of these, 41 were duplicates, bringing the total number of results to 149. The results included journal articles, article reviews, and journal editorials.

For Step 3, the inclusion criteria included the following: English articles from the past 25 years, all types of research from any country, active duty military personnel and interventions involving electrophysical agents, exercise, range of motion, strength, proprioception, functional tasks, orthotics, braces, and/or manual techniques. Exclusion criteria included: articles about amputees, traumatic brain injury, neurological injuries, blast injuries, or veterans. Of the 149 articles, all were read in their entirety by the authors, and after applying the inclusion/exclusion criteria, 31 articles were included in our review.

Articles were then summarized (Table 1), and the data was charted, as in Step 4, based on the interventions used and the return-to-duty rates (Table 2) as well as injury type and outcome measures used (Table 3). The charted data was then evaluated to determine (1) publications by country and by type; (2) scope and incidence of injury types encountered; (3) scope and prevalence of interventions used as part of physical therapy; and (4) the effectiveness of these interventions as shown by the return-to-duty rates (Step 5 ).

\section{RESULTS}

\section{Publications by country and by type}

Out of the 31 included articles in this review, 27 came from the United States, one from Thailand, two from the United Kingdom, and one was a collaborative effort from both the United States and the United Kingdom. Many of the studies reported data on troops while they were deployed abroad, and we classified them based on the nationality of the personnel included and where the publication originated. Publications included eight retrospective reviews, seven case series, seven prospective cohort studies, five randomized controlled trials, two review articles, and one literature review. These data are presented in Table 1. 
Table 1. Summary of study information and participant data

\begin{tabular}{|c|c|c|c|}
\hline Study & Type of study & $\begin{array}{l}\text { Participants' } \\
\text { nation }\end{array}$ & Information obtained from \\
\hline Islinger et al. (1998)5 & $\begin{array}{l}\text { Retrospective } \\
\text { review }\end{array}$ & United States & $\begin{array}{l}22 \text { active duty military personnel from the Walter } \\
\text { Reed Army Medical Center in the United States }\end{array}$ \\
\hline Blood et al. $(1994)^{6}$ & Case series & $\begin{array}{l}\text { United States, } \\
\text { United Kingdom }\end{array}$ & $\begin{array}{l}\text { Administrative and medical records from military } \\
\text { personnel injured in ground operations in Okinawa, } \\
\text { Korea, Vietnam, and the Falkland Islands }\end{array}$ \\
\hline Kumnerddee, W. (2009) ${ }^{7}$ & $\begin{array}{l}\text { Randomized } \\
\text { controlled trial }\end{array}$ & Thailand & $\begin{array}{l}18 \text { on-service male military personnel from the 4th } \\
\text { Battalion, 1st Regiment and king's own bodyguard } \\
\text { from Thailand }\end{array}$ \\
\hline Cullison et al. $(1998)^{8}$ & Case series & United States & $\begin{array}{l}120 \text { active duty military personnel who underwent } \\
\text { anterior cruciate ligament (ACL) reconstruction } \\
\text { from the Naval Medical Centre in the United States }\end{array}$ \\
\hline DiStasio II et al. (1994) & $\begin{array}{l}\text { Randomized } \\
\text { controlled trial }\end{array}$ & United States & $\begin{array}{l}61 \text { active duty military personnel with operatively } \\
\text { treated ankle fractures from the Naval Medical } \\
\text { Center in the United States }\end{array}$ \\
\hline Enad et al. $(2001)^{10}$ & $\begin{array}{l}\text { Retrospective } \\
\text { review }\end{array}$ & United States & $\begin{array}{l}13 \text { active military personnel who underwent } \\
\text { patellar tendon repair at a military medical center } \\
\text { in the United States }\end{array}$ \\
\hline Glebus et al. (2013) ${ }^{11}$ & $\begin{array}{l}\text { Retrospective } \\
\text { review }\end{array}$ & United States & $\begin{array}{l}45 \text { active duty military personnel who underwent } \\
\text { hip and knee arthroplasty between March } 2005 \\
\text { and June } 2008 \text { at Brooke Army Medical Center in } \\
\text { the United States }\end{array}$ \\
\hline Doukas et al. (2006) ${ }^{12}$ & Case series & United States & $\begin{array}{l}\text { A large volume of active duty military personnel } \\
\text { at the Walter Reed Army Medical Center in the } \\
\text { United States who underwent physical medicine } \\
\text { and rehabilitation (PMR) service, which was well } \\
\text { demonstrated during Operation Enduring Freedom } \\
\text { and Operation Iraqi Freedom }\end{array}$ \\
\hline Cook et al. (2013) ${ }^{13}$ & Case series & United States & $\begin{array}{l}25 \text { active duty military personnel who underwent } \\
\text { coracoclavicular ligament reconstruction at the } \\
\text { Tripler Army Medical Center in the United States } \\
\text { since } 2009\end{array}$ \\
\hline Weisel S. (2010) ${ }^{14}$ & Review article & United States & $\begin{array}{l}\text { A review of studies involving } 34,000 \text { members } \\
\text { of the US military evacuated from Iraq and } \\
\text { Afghanistan from } 2004 \text { to } 2007\end{array}$ \\
\hline Braverman SE (2002) ${ }^{15}$ & Case series & United States & US Army PMR clinics during the Persian Gulf War \\
\hline Cruser et al. (2012) ${ }^{16}$ & $\begin{array}{l}\text { Randomized } \\
\text { controlled trial }\end{array}$ & United States & $\begin{array}{l}\text { Active duty military personnel at the Madigan Army } \\
\text { Medical Center in the United States }\end{array}$ \\
\hline Gatchel et al. (2009) ${ }^{17}$ & $\begin{array}{l}\text { Randomized } \\
\text { controlled trial }\end{array}$ & United States & $\begin{array}{l}\text { Active duty US soldiers referred to Wilford Hall } \\
\text { Medical Centre and Brooke Army Medical Centre }\end{array}$ \\
\hline Cohen et al. $(2012)^{18}$ & $\begin{array}{l}\text { Literature } \\
\text { review }\end{array}$ & United States & $\begin{array}{l}\text { Troops from various countries, mainly from the } \\
\text { United States }\end{array}$ \\
\hline Withnall et al. (2006) ${ }^{19}$ & $\begin{array}{l}\text { Randomized } \\
\text { controlled trial }\end{array}$ & United Kingdom & $\begin{array}{l}\text { Royal Air Force (RAF) personnel undergoing basic } \\
\text { training in the United Kingdom (RAF Halton). }\end{array}$ \\
\hline Cohen et al. (2010) ${ }^{1}$ & $\begin{array}{l}\text { Prospective } \\
\text { cohort study }\end{array}$ & United States & $\begin{array}{l}\text { A database maintained by the Deployed Warrior } \\
\text { Medical Management Center in Germany. }\end{array}$ \\
\hline Cohen et al. (2005) ${ }^{2}$ & $\begin{array}{l}\text { Epidemiological } \\
\text { study }\end{array}$ & United States & $\begin{array}{l}162 \text { soldiers evacuated from Operation Iraqi } \\
\text { Freedom who had sustained injuries in Iraq, } \\
\text { Kuwait, or Qatar. Medical records from the } \\
\text { department of clinical investigation at Walter Reed } \\
\text { Army Medical Center in the United States or the } \\
\text { Landstuhl Regional Army Medical Center Pain } \\
\text { Clinic in Germany between March } 2003 \text { and July } \\
2004 \text {. }\end{array}$ \\
\hline
\end{tabular}


Houghton et al.

Table 1. Continued

\begin{tabular}{|c|c|c|c|}
\hline Study & Type of study & $\begin{array}{l}\text { Participants' } \\
\text { nation }\end{array}$ & Information obtained from \\
\hline Cohen et al. $(2010)^{20}$ & $\begin{array}{l}\text { Prospective } \\
\text { cohort study }\end{array}$ & United States & $\begin{array}{l}374 \text { soldiers evacuated from Afghanistan, Iraq, } \\
\text { Kuwait, Kyrgyzstan, Qatar, and so on between } \\
2004 \text { and } 2007 \text {. Data collected from the Deployed } \\
\text { Warrior Medical Management Center in Germany }\end{array}$ \\
\hline Silverwood et al. $(2012)^{21}$ & Case study & United Kingdom & $\begin{array}{l}\text { One Royal Naval Serviceman who had an } \mathrm{ACL} \\
\text { reconstruction in Belgium and returned to the } \\
\text { United Kingdom when he failed to return to } \\
\text { expected fitness levels }\end{array}$ \\
\hline Orr et al. $(2013)^{22}$ & $\begin{array}{l}\text { Prospective } \\
\text { cohort study }\end{array}$ & United States & $\begin{array}{l}15 \text { patients with acute Achilles tendon ruptures } \\
\text { between October } 2009 \text { and March } 2012 \text {. Depart- } \\
\text { ment of Orthopaedic Surgery William Beaumont } \\
\text { Army Medical Centre in the United States }\end{array}$ \\
\hline Hauret et al. (2001) 23 & $\begin{array}{l}\text { Prospective } \\
\text { Cohort Study }\end{array}$ & United States & $\begin{array}{l}\text { Soldiers in basic combat training in Fort Jackson, } \\
\text { SC, who were unable to continue training due to } \\
\text { one or more training-related injuries }\end{array}$ \\
\hline Hoppes et al. (2013) 24 & Case study & United States & $\begin{array}{l}\text { A } 29 \text {-year old male army soldier serving in Iraq } \\
\text { who underwent an acute pectoralis major tendon } \\
\text { rupture while weight training }\end{array}$ \\
\hline McCormick et al. (2014) 25 & $\begin{array}{l}\text { Prospective } \\
\text { cohort study }\end{array}$ & United States & $\begin{array}{l}42 \text { active duty soldiers with an arthroscopically } \\
\text { confirmed type II SLAP lesion, who underwent } \\
\text { repair surgery but were still unable to return to } \\
\text { active duty and who met operative failure criteria }\end{array}$ \\
\hline Shaha et al. $(2013)^{26}$ & $\begin{array}{l}\text { Retrospective } \\
\text { review }\end{array}$ & United States & $\begin{array}{l}38 \text { active duty soldiers who underwent consecu- } \\
\text { tive osteochondral allograft transplantations at the } \\
\text { Tripler Army Medical Centre in the United States }\end{array}$ \\
\hline Vachon et al. (2009) 27 & $\begin{array}{l}\text { Retrospective } \\
\text { review }\end{array}$ & United States & $\begin{array}{l}11 \text { trainees presenting with acute painless shoulder } \\
\text { weakness during navy special warfare training } \\
\text { between August } 2005 \text { and August } 2006\end{array}$ \\
\hline White and Cohen (2007) ${ }^{28}$ & $\begin{array}{l}\text { Prospective } \\
\text { cohort study }\end{array}$ & United States & $\begin{array}{l}132 \text { personnel at the Ibn Sina Pain Clinic in } \\
\text { Baghdad, Iraq, between October } 2005 \text { and } \\
\text { September } 2006 \text {. Personnel included US, British, } \\
\text { and Australian soldiers, a US contractor, and Iraqi } \\
\text { nationals }\end{array}$ \\
\hline Cross et al. $(2012)^{29}$ & $\begin{array}{l}\text { Retrospective } \\
\text { review }\end{array}$ & United States & $\begin{array}{l}115 \text { soldiers who sustained battle-related type III } \\
\text { open tibia fractures }\end{array}$ \\
\hline McCallum et al. $(2014)^{30}$ & $\begin{array}{l}\text { Retrospective } \\
\text { review }\end{array}$ & United States & $\begin{array}{l}46 \text { military personnel who underwent elective } \\
\text { fasciotomies between } 2007 \text { and } 2011 \text { at a Tertiary } \\
\text { Military Medical Center in the United States }\end{array}$ \\
\hline Teyhen (1999) 31 & Review article & United States & $\begin{array}{l}550 \text { military personnel who required physical } \\
\text { therapy in a military medical center in Hungary } \\
\text { between December } 1996 \text { and March } 1997\end{array}$ \\
\hline Prahinski et al. (1996) ${ }^{32}$ & $\begin{array}{l}\text { Retrospective } \\
\text { review }\end{array}$ & United States & $\begin{array}{l}10 \text { military personnel who have undergone surgery } \\
\text { for paresis of anterior and/or lateral leg musculature } \\
\text { at the Walter Reed Army Medical Center in the } \\
\text { United States since } 1985\end{array}$ \\
\hline Goss et al. (2009) 33 & $\begin{array}{l}\text { Prospective } \\
\text { cohort study }\end{array}$ & United States & $\begin{array}{l}90 \text { military personnel participating in a functional } \\
\text { training program following injury rehabilitation from } \\
\text { August } 2006 \text { to December } 2008\end{array}$ \\
\hline Booth-Kewley et al. (2014) & $\begin{array}{l}\text { Prospective } \\
\text { cohort study }\end{array}$ & United States & $\begin{array}{l}134 \text { US Marines who were surveyed at baseline } \\
\text { following an musculoskeletal injury (back, knee, } \\
\text { or shoulder) and again one year following } \\
\text { rehabilitation }\end{array}$ \\
\hline
\end{tabular}

Note: The number of personnel was not included for studies that did not state this information. 
Table 2. Summary of interventions used by each study and return-to-duty rates




Table 2. Continued

\begin{tabular}{|c|c|c|}
\hline Type of intervention & Studies using intervention & Return-to-active-duty rates \\
\hline \multicolumn{3}{|l|}{ Modalities } \\
\hline - Electrical stimulation & Vachon et al. $(2009)^{27}$ & $100 \%$ (11/11 subjects, mean 21 weeks) \\
\hline - Ultrasound & Cohen et al. $(2005)^{2}$ & $2 \%$ of 49 patients for whom data was available \\
\hline - Heat & Vachon et al. (2009) 27 & $100 \%$ (11/11 subjects, mean 21 weeks) \\
\hline - Cryotherapy & Hoppes et al. $(2013)^{24}$ & $100 \%$ (1/1 subjects, in 6 months) \\
\hline & Vachon et al. (2009) 27 & $100 \%$ (11/11 subjects, mean 21 weeks) \\
\hline - Acupuncture & Kumnerddee $(2009)^{7}$ & None stated \\
\hline- & Cohen et al. $(2005)^{2}$ & $2 \%$ of 49 patients for whom data was available \\
\hline $\begin{array}{l}\text { Manual therapy } \\
\text { - Soft Tissue Release/Massage }\end{array}$ & $\begin{array}{l}\text { Kumnerddee }(2009)^{7} \\
\text { Cohen et al. }(2005)^{2}\end{array}$ & $\begin{array}{l}\text { None stated } \\
2 \% \text { of } 49 \text { patients in whom data was available }\end{array}$ \\
\hline - Mobilizations & Cruser et al. $(2012)^{16}$ & None stated \\
\hline - Manipulations & Cruser et al. (2012)) $)^{16}$ & None stated \\
\hline - Muscle energy & Cruser et al. (2012)) $)^{16}$ & None stated \\
\hline $\begin{array}{l}\text { Bracing/Orthoses/Orthotics } \\
\text { - Spinal orthosis }\end{array}$ & $\begin{array}{l}\text { Islinger et al. }(1998)^{5} \\
\text { Enad et al. }(2001)^{10}\end{array}$ & $\begin{array}{l}22 \% \text { ( } 2 / 9 \text { subjects) } \\
92 \% \text { ( } 12 / 13 \text { subjects, mean } 13 \text { months) }\end{array}$ \\
\hline - Orthotics, orthoses, ankle-foot orthoses & $\begin{array}{l}\text { Braverman (2002) }{ }^{15} \\
\text { Withnall et al. }(2006)^{19} \\
\text { Prahinski et al. }(1996)^{32} \\
\text { DiStasio II et al. }(1994)^{9}\end{array}$ & $\begin{array}{l}\text { None stated } \\
\text { None stated } \\
42.8 \% \text { ( } 3 / 7 \text { subjects) } \\
\text { Group 1: } 100 \% \text { in } 6.2 \text { months } \\
\text { Group } 2: 100 \% \text { in } 5.8 \text { months }\end{array}$ \\
\hline - Removable short-leg orthosis & Orr et al. $(2013)^{22}$ & $100 \%$ (15/15 patients, mean 16.7 months) \\
\hline - Non-weight bearing $20^{\circ}$ equinus splint & Orr et al. $(2013)^{22}$ & $100 \%$ (15/15 patients, mean 16.7 months) \\
\hline $\begin{array}{l}\text { - Removable walking boot with } 1.5^{\prime \prime} \\
\text { heel lift }\end{array}$ & Hoppes et al. $(2013)^{24}$ & $100 \%$ ( $1 / 1$ subjects, in 6 months) \\
\hline - Sling/shoulder immobilizer & $\begin{array}{l}\text { McCormick et al. }(2014)^{25} \\
\text { Vachon et al. }(2009)^{27}\end{array}$ & $\begin{array}{l}81 \% \text { (34/42 subjects, in } 3 \text { months) } \\
100 \% \text { ( } 11 / 11 \text { subjects, mean } 21 \text { weeks) }\end{array}$ \\
\hline
\end{tabular}

Notes: Studies not included in this table did not state interventions. These studies include Blood et al. (2006), ${ }^{6}$ Weisel (2010), ${ }^{14}$ Cohen et al. (2010), ${ }^{1}$ Cohen et al. (2010), ${ }^{20}$ Cross et al. (2012), ${ }^{29}$ Cohen et al. (2012), ${ }^{18}$ McCallum et al. (2014), ${ }^{30}$ Booth-Kewley et al. (2014), ${ }^{34}$ Teyhen (1999). ${ }^{31}$ Of note is one study that stated that $14 \%$ of service members returned to duty and that $88 \%$ of these returned with no physical limitations. ${ }^{20}$ Some studies that did not state returnto-duty rates did mention that there was increased functional improvements made in the group that received physical therapy, ${ }^{17}$ that significant increases occurred in all functional tests used, ${ }^{33}$ and that return-to-duty rates were based on the assessment, management, and disposition of the injured patient. ${ }^{12}$

\section{Physical therapy interventions}

Articles described physical therapy interventions as single treatments or combinations of range of motion (ROM) exercises, stretching, strengthening exercises, aerobic exercise, manual therapy, modalities, or bracing/ orthotics/orthoses. The frequency of inclusion of each type of treatment was as follows: 13 articles on strengthening (manual muscle testing, rehabilitation-specific exercises, functional task training, or unspecified exercise); 10 articles on bracing/orthotics/orthoses (spinal orthoses, ankle-foot orthoses, removable short-leg orthoses, un- designated orthoses, orthotics, ankle splints, walking boots, and shoulder immobilizer/sling); nine articles on ROM and continuous passive motion; four articles on modalities (electrical stimulation, ultrasound, heat, cryotherapy, and acupuncture); three articles on aerobic exercise defined as either general conditioning or physical fitness training; 3 articles on manual therapy (soft tissue release/massage, mobilizations, manipulations, and muscle energy techniques); and one article on stretching. These data are presented in Table 2. 
Table 3. Summary of type of injury and the number of studies using specific outcome measures

\begin{tabular}{|c|c|c|}
\hline Type of injury & Outcome measures & $\begin{array}{l}\text { Number of studies using } \\
\text { this outcome measure }\end{array}$ \\
\hline $\begin{array}{l}\text { Failed superior labrum } \\
\text { anterior or posterior repairs }\end{array}$ & $\begin{array}{l}\text { - American shoulder and elbow surgeons shoulder score } \\
\text { - Western Ontario shoulder instability index } \\
\text { - Single assessment numeric evaluation } \\
\text { - Range of motion (ROM) for flex, Abd and R/ER at } 0 \text { and } \\
90 \text { degrees }\end{array}$ & McCormick et al. (2014)25 \\
\hline Complete ACL tear & $\begin{array}{l}\text { - Lysholm score } \\
\text { - Subjective patient scale (0-10) } \\
\text { - KT1000 evaluation } \\
\text { - Pivot shift } \\
\text { - ROM } \\
\text { - Lachman's scores }\end{array}$ & $\begin{array}{l}\text { Cullison et al. (1998); } \\
\text { Silverwood et al. }(2012)^{21}\end{array}$ \\
\hline Acute Achilles tendon rupture & $\begin{array}{l}\text { - American Orthopaedic Foot and Ankle Society score } \\
\text { - Visual analogue scale (VAS) } \\
\text { - ROM }\end{array}$ & Orr et al. $(2013)^{22}$ \\
\hline $\begin{array}{l}\text { Overuse trauma stress } \\
\text { fracture or other }\end{array}$ & $\begin{array}{l}\text { - US Army fitness test } \\
\text { - Score (appropriate to the week of training a soldier } \\
\text { would be returning to) } \\
\text { - Time on limited duties }\end{array}$ & Hauret et al. $(2001)^{23}$ \\
\hline $\begin{array}{l}\text { Acute pectoralis major } \\
\text { tendon rupture }\end{array}$ & $\begin{array}{l}\text { - VAS } \\
\text { - Resumption of full military duties } \\
\text { - Completion of } 50 \text { consecutive pushups without issue }\end{array}$ & Hoppes et al. $(2013)^{24}$ \\
\hline Myofascial back pain & $\begin{array}{l}\text { - Short-form McGill pain questionnaire } \\
\text { - } 100 \mathrm{~mm} \text { VAS } \\
\text { - Summation of pain pressure threshold in each trigger } \\
\text { point measured by a pressure algometer }\end{array}$ & Kumnerddee, W. (2009) ${ }^{7}$ \\
\hline Spine fractures & $\begin{array}{l}\text { - Correlation between initial treatment and physical job } \\
\text { demands } \\
\text { - RTD rates }\end{array}$ & Islinger et al. $(1998)^{5}$ \\
\hline Ankle fractures & $\begin{array}{l}\text { - “Figure-of-eight” tape method } \\
\text { - Gross manual muscle testing } \\
\text { - Single-stance toe raises, } \\
\text { - ROM } \\
\text { - Platform jump and cutting ability } \\
\text { - Subjective score } \\
\text { - Maryland foot score }\end{array}$ & DiStasio II et al. (1994) ${ }^{9}$ \\
\hline Chondral injuries (knee) & $\begin{array}{l}\text { - Knee injury and osteoarthritis outcome score } \\
\text { - Single assessment numeric scale } \\
\text { - Return to full duty in military occupational specialty }\end{array}$ & Shaha et al. (2013) ${ }^{26}$ \\
\hline Patellar tendon repairs & $\begin{array}{l}\text { - Lysholm scores } \\
\text { - Tegner activity scores } \\
\text { - Circumferential thigh girth and active extension deficit } \\
\text { - Insall-Salvati ratio } \\
\text { - Merchant angle } \\
\text { - Function-based on subjective report of pain and level } \\
\text { - } \text { of activity regained } \\
\text { - Patellofemoral compression tenderness } \\
\text { - ROM }\end{array}$ & Enad et al. $(2001)^{10}$ \\
\hline $\begin{array}{l}\text { Total hip or knee joint } \\
\text { arthroplasty }\end{array}$ & $\begin{array}{l}\text { - Short musculoskeletal function assessment } \\
\text { - Deployment specific questionnaire }\end{array}$ & Glebus et al. (2013) ${ }^{11}$ \\
\hline
\end{tabular}


Houghton et al.

Table 3. Continued

\begin{tabular}{|c|c|c|}
\hline Type of injury & Outcome measures & $\begin{array}{l}\text { Number of studies using } \\
\text { this outcome measure }\end{array}$ \\
\hline $\begin{array}{l}\text { Brachial plexus injuries } \\
\text { (Acute, Painless Shoulder } \\
\text { Weakness) }\end{array}$ & $\begin{array}{l}\text { - Manual muscle testing with Medical Research Council } \\
\text { criteria } \\
\text { - Pre-injury amount of timed two-minute push ups and } \\
\text { pull ups }\end{array}$ & Vachon et al. (2009) 27 \\
\hline $\begin{array}{l}\text { Coracoacromial ligament } \\
\text { reconstructions }\end{array}$ & $\begin{array}{l}\text { - Conoid ratio } \\
\text { - Trapezius ratio } \\
\text { - Radiographs with picture archiving and communication } \\
\text { system for measurements }\end{array}$ & Cook et al. $(2013)^{13}$ \\
\hline Acute low back pain & $\begin{array}{l}\text { - Quadruple VAS } \\
\text { - Roland Morris Disability Questionnaire } \\
\text { - Short-form Health Survey } 36\end{array}$ & Cruser et al. (2012) ${ }^{16}$ \\
\hline Unspecified MSK disorders & $\begin{array}{l}\text { - VAS } \\
\text { - Dallas Pain and Disability Questionnaire } \\
\text { - Owestry Disability Questionnaire } \\
\text { - Short-form Health Survey } 36 \\
\text { - Beck Depression Inventory II } \\
\text { - Multidimensional pain inventory } \\
\text { - Fear avoidance beliefs questionnaire } \\
\text { - Functional capacity evaluation }\end{array}$ & $\begin{array}{l}\text { Gatchel et al. (2009) }{ }^{17} \\
\text { Booth-Kewley et al. } \\
(2014)^{34}\end{array}$ \\
\hline $\begin{array}{l}\text { MSK and connective tissue } \\
\text { disorders }\end{array}$ & - RTD rates after injury within two weeks & Cohen et al. $(2010)^{1}$ \\
\hline Muscle pain & - VAS pain scores (0-10 scale) & Cohen et al. $(2005)^{2}$ \\
\hline $\begin{array}{l}\text { Soft tissue injury } \\
\text { (non-muscular) }\end{array}$ & - RTD rates & \\
\hline \multicolumn{3}{|l|}{ Sacroiliac joint pain } \\
\hline \multicolumn{3}{|l|}{ Plantar fasciitis } \\
\hline \multicolumn{3}{|l|}{ Low back pain } \\
\hline \multicolumn{3}{|l|}{ Arm and leg injuries } \\
\hline \multicolumn{3}{|l|}{ Neck pain } \\
\hline Neck pain & - RTD rates & Cohen et al. $(2010)^{20}$ \\
\hline $\begin{array}{l}\text { Paresis of anterior and lateral } \\
\text { compartments of the leg }\end{array}$ & $\begin{array}{l}\text { - Functional status } \\
\text { - RTD rates } \\
\text { - Ankle ROM }\end{array}$ & Prahinski et al. (1996) ${ }^{32}$ \\
\hline
\end{tabular}

\section{Return-to-duty rates}

Return-to-duty rates varied highly between studies, as did the length of time for the treatment protocols in the studies. Return-to-duty rates were compared in the context of the interventions used in the study wherever the information was available. Some studies did not provide return-to-duty rates. Strengthening interventions had return-to-duty rates ranging from 29 to 100 per cent over a span of 3-13 months; bracing, orthotics, and orthoses-based interventions ranged from 22 to 100 per cent return to duty over 3-16.7 months; ROM interventions ranged from 29 to 100 per cent return to duty over 3-16.7 months; modalities ranged from 2 to $100 \%$ return to duty over 21-24 weeks; aerobic exercise ranged from 86 to $100 \%$ return to duty over 6-20.9 months; manual therapy ranged from 2 per cent return to duty with no time frame given; and stretching had a 100 per cent return to duty rate over 21 weeks. These data are presented in Table 2.

\section{Injury types}

The data revealed trends based on anatomic structure as well as by injury type. The following number of articles described injuries based on anatomic structure: 2 on the neck; 4 on the shoulder; 4 on the back/spine; 1 on the hip; 5 on the knee; 2 on the leg; and 3 on the ankle/ foot. In the same pool of articles, 8 articles were found on tendon, ligament, or labral pathology; 4 on fractures; 
3 on articular pathology; 4 on myofascial pain; 2 on paresis; 2 on overuse injuries; and 3 on non-specific musculoskeletal injuries. It should be noted that some articles described injuries to multiple areas and/or multiple injury types and were therefore counted multiple times in the preceding description. There was a wide array of outcome measures used between studies for each injury type. These data are presented in Table 3.

\section{DISCUSSION}

Musculoskeletal injuries are the most common reason for medical evacuation in the military, and they account for more than half of hospital stays after injury. ${ }^{1,2,14}$ Overall, the most commonly injured body parts in soldiers appear to be the shoulder, back, knee, ankle, and foot. Given the weight of a soldier's equipment configuration, which may reach in excess of 100 pounds, and that they are required to repeatedly hike, run, jump, lift, carry, and manoeuvre tactically while supporting this added weight, it follows logically that these joints would be the most loaded and, therefore, develop pathology. Hauret et al. ${ }^{23}$ report that 44 per cent of the soldiers admitted to the rehabilitation program at Fort Jackson had sustained an overuse injury during basic training, and a further 39 per cent had sustained a stress fracture. Injuries negatively affect military training and operational output, and treatment is often time-consuming and difficult. ${ }^{19}$ Some studies have examined how specialized equipment may mitigate overuse injuries with mixed results. Withnall et al. ${ }^{19}$ found that shock-absorbing insoles did not provide any significant improvements over standard-issue insoles in lower extremity injury rates during Royal Air Force basic training. Another study mentioned that the use of orthotics, orthoses, and ankle-foot orthoses do help return to duty, but it did not include subject numbers to support the finding. ${ }^{15}$ Injury prevention through equipment modification remains a promising field of research for the military, one that is arguably clinically and economically justified. ${ }^{19}$ In addition, continued instruction on proper lifting techniques remains necessary given the high frequency of loading and unloading support vehicles in military duties. ${ }^{31}$ The use of ultrasound, massage therapy, and acupuncture as interventions yielded poor returnto-duty rates with a 2 per cent return-to-duty rate or, in the case of one acupuncture article by Kumnerddee, ${ }^{7}$ no return-to-duty rates reported at all. ${ }^{2,7}$ In contrast, the use of electrical stimulation, heat, and cryotherapy yielded promising return-to-duty rates with 100 per cent of individuals returning following the use of these treatments. ${ }^{24,27}$

The most widely used interventions post-injury are immobilization/bracing of unstable or weakened structures, ROM exercises, and strengthening weakened musculature followed by a graded return to functional tasks. ${ }^{5,8-11,13,15,17,19,22-8,32,33}$ Though the associated return-to-duty rates span a large range, many are approximately $80 \%$ and above for soldiers returning to full duties at an average time of 3-6 months. $^{9-}$ $11,13,18,21,22,24,25,27,28,30$ In many cases, these interventions were used in conjunction with traditional military medical care that was largely focused on medication prescription ${ }^{6,16,17}$ or surgery. ${ }^{5,8-11,13,26}$ Doukas et al. ${ }^{12}$ used a combined rehabilitation therapy approach involving a minimum of three hours of therapy a day from physiotherapy, occupational therapy, speech language pathology, social work or psychology, and medical treatment in a rehabilitation unit; however, the authors did not mention return-to-duty rates or the specifics of each intervention. This combined therapy was used to treat a variety of conditions including non-weight bearing fractures, orthopaedic extremity injuries, post-surgical rehabilitation, and MSK injuries involving the neck, back, shoulder, and knee as a result of repetitive trauma and aggravation from the demands and stress of combat. These injuries prevented soldiers from continuing their duties, and, as a result, they were transferred to they rehabilitation facility where they received care from a variety of different health care professionals. The main goal of this facility was to return soldiers back to duty as soon as possible. Each health care specialist was required to document a clear and concise treatment plan for each soldier. If a soldier would not be returning directly to duty, they would only be granted a 30-day convalescent leave to their demobilization site before returning. All physical and occupational therapy referrals for demobilization sites were required to include a frequency and time limit for continuing treatment before returning to duty. ${ }^{12}$ Using a rehabilitation team to care for injured military personnel may allow for a better understanding of the injuries and what is required for soldiers to return to duty from a variety of health care perspectives.

While the types of interventions from each study remained mostly consistent (that is, ROM, strengthening, immobilization, and so on), protocols varied significantly between studies, even for similar injuries, in the number of interventions used and the duration of intervention. (Table 3). It may be, however that the sooner a 
rehabilitation program is begun post-surgery, the better the outcomes for returning to duty. ${ }^{9}$ DiStasio II et al. ${ }^{9}$ initiated a physical therapy program for the post-operative management of ankle fractures. One group started immediately, and the other group started after six weeks. The immediate group returned to duty in less time 5.8 months - compared to the group who started later and returned in 6.2 months. This finding stresses the importance of facilitating physical therapy and structured rehabilitation programs immediately post-surgery, as it allows soldiers to recovery and return to duty sooner. It follows, then, that quick rehabilitation post-injury may yield similar results. Widespread use of structured rehabilitation programs, as in Hauret et al. ${ }^{23}$ (a program dedicated to recovering soldiers after basic combat training injuries who meet set criteria for intake and discharge, which allows training units to focus on training missions), may serve to produce a consistent percentage of soldiers returned to active duty and help better determine criteria indicative of a successful return to duty. Goss et al. ${ }^{33}$ developed a training protocol meant to further improve functionality of soldiers who have recently completed rehabilitation for any MSK injury, which included dynamic warm ups, varying gross functional strengthening exercises, focused core strengthening, and a cool down. Most studies examined in this review included functional training as part of their interventions, although consideration may be given to a protocol such as this to maximize physical ability and prevent re-injury following return to duty.

In the US, officers and senior enlisted personnel are seen to be more likely to return to duty than junior service members ${ }^{1,5,8,11,29,30}$ due to the relatively lower levels of physical stress that are associated with these positions. Similarly, soldiers in combat arms specialties are less likely to return to active duty post-injury due to the higher physical stresses placed on them in comparison to those in combat support specialties. ${ }^{26}$ Shaha et al. ${ }^{26}$ found that soldiers in the navy or marine corps were more likely to return to duty than those in the army or air force. ${ }^{26}$ Cohen et al. ${ }^{20}$ also found that marines were over four times more likely to return to their units compared to army soldiers. One possible explanation is the allowance of substitute physical fitness tests with fewer high-load activities, as is found in the navy, which allows more soldiers to return to duty despite some lasting performance degradation. ${ }^{26}$ It has also been hypothesized that return-to-duty rates are better in military branches where activity modification has a significant impact on duty status. ${ }^{1,8,26}$ For example, the marine corps allows no substitution to the mandated fitness tests regardless of injury, and marines must return to full, unrestricted duty within 6-12 months or face expulsion from the corps. ${ }^{26}$ Neither the army nor air force have such aggressive guidelines regarding return following a brief period of limited duty status, and this may explain, in part, why return-to-duty rates are lower than those of the navy and marines. ${ }^{26}$

Rehabilitation protocols should recognize these disparities and allow appropriate recovery time for an individual soldier based on rank, branch of service, and military occupational specialty (MOS) as well as injury type and severity. Enad et al. ${ }^{10}$ ensured that active duty military personnel were able to completely perform the physical requirements for their MOS in addition to being able to run 1.5 miles as a physical readiness test before returning to duty. This assists in creating realistic expectations for recovery, which also appears to be a predictive factor of return-to-duty success. ${ }^{34}$ Ensuring that military personnel are able to perform their regular duties is important for return-to-duty rates. Glebus et al. ${ }^{11}$ looked at a soldier's ability to perform various deployment-related activities in a combat zone and assessed functional tasks in addition to physical therapy to determine return-to-duty rates following total hip and knee arthroplasty. As a result, there was a high return-toduty rate $(86 \%)$ with an additional high deployment rate $(70 \%)$ back to the combat zone after sustaining a joint injury that required surgery. ${ }^{11}$ Assessing the ability of military personnel to complete these specific tasks may lead to higher and more successful return-to-duty rates.

Limitations in this review include the overwhelming amount of data from the United States and the relative paucity of information from other armed forces worldwide. While this calls into question the generalizability of the findings of this review among nations, it can be concluded that heavily loaded and/or repeated tasks comprise a large portion of military duty regardless of nationality. Therefore, similar injuries are likely to be seen and, given the large pool of healthcare data accessible globally, treated with similar interventions. Another limitation is that the variability in interventions and outcome measures used between studies makes it difficult to determine concrete criteria for the safe return to active duty for any given injury type. We were, however, able to determine trends among the prevalent injury types and treatment approaches in military populations. Future 
studies should focus on standardized interventions and outcome measures that take into consideration the type of injury, completion of functional tasks, and physical requirements for MOS and, furthermore, how successful these interventions are at returning personnel safely to active duty. This information would allow future researchers to determine what functional criteria must be met to ensure safe return to duty following injury.

\section{CONCLUSION}

Overall, there is a currently a paucity of literature on interventions and functional outcomes that can be used to guide rehabilitation of various MSK injuries in a military population and to determine the criteria to facilitate successful return to duty. The existing literature does provide information on prevalent injury types, varying treatment approaches and return-to-duty rates among military populations. The use of standardized protocols in treating military injuries and taking into consideration the physical requirements for each MOS may assist in creating a set of criteria effective at determining readiness for a safe return to active duties. Until then, a graded return to functional tasks should be used to return injured soldiers to full functional status.

\section{REFERENCES}

1. Cohen SP, Brown C, Kurihara C, et al. Diagnoses and factors associated with medical evacuation and return to duty for service members participating in Operation Iraqi Freedom or Operation Enduring Freedom: a prospective cohort study. Lancet. 2010;375(9711):301-9. http://dx.doi.org/10.1016/S0140-6736(09)61797-9. Medline:20109957

2. Cohen SP, Griffith S, Larkin TM, et al. Presentation, diagnoses, mechanisms of injury, and treatment of soldiers injured in Operation Iraqi Freedom: an epidemiological study conducted at two military pain management centers. Anesth Analg. 2005;101(4):1098-103. http://dx.doi.org/10.1213/01.ane.0000169332.45209.cf. Medline: 16192528

3. Arksey H, O’Malley L. Scoping studies: Towards a methodological framework. Int J Soc Res Methodol. 2005;8(1):19-32. http://dx.doi.org/10.1080/ 1364557032000119616.

4. Levac D, Colquhoun H, O’Brien KK. Scoping studies: advancing the methodology. Implement Sci. 2010;5(1):69. http://dx.doi.org/10.1186/1748-5908-569. Medline:20854677

5. Islinger RB, Kuklo TR, Polly DW Jr. Spine fractures in active duty soldiers and their return to duty rate. Mil Med. 1998;163(8):536-9. Medline:9715617

6. Blood CG, Gauker ED, Jolly R, et al. Comparisons of casualty presentation and admission rates during various combat operations. Mil Med. 1994;159(6):457-61. Medline:7984306

7. Kumnerddee W. Effectiveness comparison between Thai traditional massage and Chinese acupuncture for myofascial back pain in Thai military personnel: a preliminary report. J Med Assoc Thai. 2009;92(Suppl 1):S117-23. Medline:21299184

8. Cullison TR, O’Brien TJ, Getka K, et al. Anterior cruciate ligament reconstruction in the military patient. Mil Med. 1998;163(1):17-19. Medline:9465566

9. DiStasio AJ II, Jaggears FR, DePasquale LV, et al. Protected early motion versus cast immobilization in postoperative management of ankle fractures. Contemp Orthop. 1994;29(4):273-7. Medline:10150249

10. Enad JG, Loomis LL. Primary patellar tendon repair and early mobilization: results in an active-duty population. J South Orthop Assoc. 2001;10(1):17-23. Medline: 12132838

11. Glebus GP, Feather TW, Hsu JR, et al. Return to duty and deployment after major joint arthroplasty. J Arthroplasty. 2013;28(8):1270-3. http://dx.doi.org/10.1016/ j.arth.2013.02.028. Medline:23830502

12. Pasquina PF, Gambel J, Foster LS, et al, and the Physical Medicine and Rehabilitation Service. Process of care for battle casualties at the Walter Reed Army Medical Center: Part III. Physical medicine and rehabilitation service. Mil Med. 2006;171(3):206-8. http://dx.doi.org/ 10.7205/MILMED.171.3.206. Medline:16602516

13. Cook JB, Shaha JS, Rowles DJ, et al. Clavicular bone tunnel malposition leads to early failures in coracoclavicular ligament reconstructions. Am J Sports Med. 2013;41(1):142-8. http://dx.doi.org/10.1177/ 0363546512465591. Medline:23139253

14. Weisel S. Back pain in the larger spectrum of wartime injuries. Black Letter. 2010;25(3):26. http://dx.doi.org/ 10.1097/01.BACK.0000369346.08534.a8.

15. Braverman SE. Orthotics for the fighting force. Phys Med Rehabil Clin N Am. 2002;13(1):159-73. http:// dx.doi.org/10.1016/S1047-9651(03)00076-7. Medline: 11878080

16. Cruser A, Maurer D, Hensel K, et al. A randomized, controlled trial of osteopathic manipulative treatment for acute low back pain in active duty military personnel. J Manual Manip Ther. 2012;20(1):5-15. http:// dx.doi.org/10.1179/2042618611Y.0000000016. Medline:23372389

17. Gatchel RJ, McGeary DD, Peterson A, et al. Preliminary findings of a randomized controlled trial of an interdisciplinary military pain program. Mil Med. 2009;174(3):270-7. http://dx.doi.org/10.7205/ MILMED-D-03-1607. Medline:19354091

18. Cohen SP, Gallagher RM, Davis SA, et al. Spine-area pain in military personnel: a review of epidemiology, etiology, diagnosis, and treatment. Spine J. 2012;12(9):833-42. http://dx.doi.org/10.1016/ j.spinee.2011.10.010. Medline:22100208 
19. Withnall R, Eastaugh J, Freemantle N. Do shock absorbing insoles in recruits undertaking high levels of physical activity reduce lower limb injury? A randomized controlled trial. J R Soc Med. 2006;99(1):32-7. http:// dx.doi.org/10.1258/jrsm.99.1.32. Medline:16388054

20. Cohen SP, Kapoor SG, Nguyen C, et al. Neck pain during combat operations: an epidemiological study analyzing clinical and prognostic factors. Spine (Phila Pa 1976). 2010;35(7):758-63. Medline:20228712

21. Silverwood R, Gordon JB, Baron R, et al. Cyclops lesions in military personnel: a reason for delayed return to fitness post anterior cruciate ligament reconstruction. J R Nav Med Serv. 2012;98(3):3-5. Medline:23311235

22. Orr JD, McCriskin B, Dutton JR. Achillon mini-open Achilles tendon repair: early outcomes and return to duty results in U.S. military service members. J Surg Orthop Adv. 2013;22(1):23-9. http://dx.doi.org/ 10.3113/JSOA.2013.0023. Medline:23449051

23. Hauret KG, Shippey DL, Knapik JJ. The physical training and rehabilitation program: duration of rehabilitation and final outcome of injuries in basic combat training. Mil Med. 2001;166(9):820-6.

Medline: 11569449

24. Hoppes CW, Ross MD, Moore JH. Undetected pectoralis major tendon rupture in a patient referred to a physical therapist in a combat environment: a case report. Phys Ther. 2013;93(9):1225-33. http://dx.doi.org/10.2522/ ptj.20120141. Medline:23086408

25. McCormick F, Nwachukwu BU, Solomon D, et al. The efficacy of biceps tenodesis in the treatment of failed superior labral anterior posterior repairs. Am J Sports Med. 2014;42(4):820-5. http://dx.doi.org/10.1177/ 0363546513520122. Medline:24519182

26. Shaha JS, Cook JB, Rowles DJ, et al. Return to an athletic lifestyle after osteochondral allograft transplantation of the knee. Am J Sports Med. 2013;41(9):20839. http://dx.doi.org/10.1177/0363546513494355. Medline:23841992

27. Vachon T, Rosenthal M, Dewing CB, et al. Acute painless shoulder weakness during high-intensity athletic training. Am J Sports Med. 2008;37(1):175-80. http:// dx.doi.org/10.1177/0363546508328101.

Medline: 19124836

28. White RL, Cohen SP. Return-to-duty rates among coalition forces treated in a forward-deployed pain treatment center: a prospective observational study. Anesthesiology. 2007;107(6):1003-8. http://dx.doi.org/ 10.1097/01.anes.0000290605.55736.e1.

Medline: 18043069

29. Cross JD, Stinner DJ, Burns TC, et al, and the Skeletal Trauma Research Consortium (STReC). Return to duty after type III open tibia fracture. J Orthop Trauma. 2012;26(1):43-7. http://dx.doi.org/10.1097/BOT. 0b013e31821c0ec1. Medline:21885998

30. McCallum JR, Cook JB, Hines AC, et al. Return to duty after elective fasciotomy for chronic exertional compartment syndrome. Foot Ankle Int.
2014;35(9):871-5. http://dx.doi.org/10.1177/ 1071100714539661. Medline:25049368

31. Teyhen DS. Physical therapy in a peacekeeping operation: Operation Joint Endeavor/Operation Joint Guard. Mil Med. 1999;164(8):590-4. Medline:10459271

32. Prahinski JR, McHale KA, Temple HT, et al. Bridle transfer for paresis of the anterior and lateral compartment musculature. Foot Ankle Int. 1996;17(10):61519. http://dx.doi.org/10.1177/107110079601701005. Medline:8908487

33. Goss DL, Christopher GE, Faulk RT, et al. Functional training program bridges rehabilitation and return to duty. J Spec Oper Med. 2009;9(2):29-48. Medline: 19813517

34. Booth-Kewley S, Schmied EA, Highfill-McRoy RM, et al. A prospective study of factors affecting recovery from musculoskeletal injuries. J Occup Rehabil. 2014;24(2):287-96. http://dx.doi.org/10.1007/s10926013-9456-7. Medline:23797182

\section{AUTHOR INFORMATION}

Nadine Houghton, MScPT, HBA Kin, is an alumnus of both Queen's University and Wilfrid Laurier University. She is a practising physiotherapy resident with an interest in orthopaedics and athletic injuries. She developed an interest in military and veteran health because of her grandfather, a Second World War veteran.

Jared Maynard, MScPT, HBSc Kin, CSCS is an alumnus of both Queen's University and McMaster University. He is a practising physiotherapy resident with a special interest in sports medicine, orthopaedics, as well as strength and conditioning. He has interned with Canadian Institute for Military and Veteran Health Research, helping its efforts to benefit veterans and their families.

Alice Aiken, $\mathrm{PhD}, \mathrm{MSc}, \mathrm{BScPT}, \mathrm{BSc}$ is an associate professor and the head of the Physical Therapy Program at the School of Rehabilitation Therapy at Queen's University in Kingston, Canada. She does health services/health policy research in the area of veteran health. She is the scientific director of the Canadian Institute for Military and Veterans Health Research.

\section{COMPETING INTERESTS}

None declared.

This article has been peer reviewed.

\section{CONTRIBUTORS}

All authors conceived, designed researched and drafted the manuscript and approved the final version submitted for publication.

\section{FUNDING}

None declared. 